\title{
Glycaemic index: challenges in translating concept to practice
}

\author{
SV Madhu ${ }^{1}$
}

Published online: 27 October 2017

(C) Research Society for Study of Diabetes in India 2017

Dietary prescribing in diabetes mellitus based on glycaemic index (GI) of foods has always been an attractive concept. Food choices with lower GI are associated with lower glucose responses after consumption and hence would be the preferred choices in patients with diabetes. While this idea has been very promising, the real challenge over the years has been its implementation in day to day practice. Several issues like validity and reproducibility of GI measurements, factors affecting glycaemic responses, differences when consumed as single foods or in mixed meals and amount of absorbable/ digestable carbohydrate need consideration besides other methodological concerns.

The concept of GI has evolved over time. When it was first proposed [1], it measured the glycaemic response of a test food as a percentage of the glycaemic response of a reference food containing a similar amount of carbohydrate. The reference food has traditionally been glucose or white bread. Even this lead to variability of GI values depending on whether it was glucose or white bread that was the reference. Individual foods have been tested and categorized as low, medium or high GI foods based on their glycaemic responses [2] when consumed alone. However, these values lose significance when the foods are consumed as part of a mixed meal [3].

The concept of GI does not take into account the amount of carbohydrate consumed even though this is a major determinant of the glycaemic response. This lead to the extension of the concept of GI to glycaemic load (GL) which is the product of GI and the total amount of carbohydrate consumed [4]. GL

SV Madhu

drsvmadhu@gmail.com

1 Department of Endocrinology, Centre for Diabetes, Endocrinology and Metabolism, University College of Medical Sciences and GTB Hospital, Delhi, India gives a fair idea of the glucose load or burden that results from the ingestion of a carbohydrate containing meal. A high GI food can have a low GL if the portion size consumed is small and a low GI food can have a high GL if the serving size is big.

It has also been realized that all carbohydrate in food is not available for conversion to glucose and hence only the amount of "available" carbohydrate also called glycaemic carbohydrate and not total carbohydrate should be used for calculation of GI. What constitutes available carbohydrate has also been a subject of debate and the consensus view is that the undigestable carbohydrate which includes the fibre and all resistant starch is taken as unavailable [5].

There are several limitations to the usefulness of GI and GL. Studies suggest that GI could be affected by many factors including the amounts of other nutrients such as fat, protein and fibre, structure of the carbohydrate, particle size, food form, food processing and cooking method [5]. These factors lead to a lot of intra and inter individual variability of GI values and GL making these estimates less reliable and raise questions about their validity [6]. The rates of digestion of carbohydrates also vary with health status, race, gender and underlying insulin resistance all of which can affect GI [5]. Similarly, methodological differences such as the amount of tested food which contains $50 \mathrm{~g}$ of carbohydrate (available, absorbable, digestable), method of glucose measurement, time of day when the test was performed and the method of calculation of glucose response also affect GI values further complicating interpretation and limiting their value for day to day use [7].

In vitro models for estimation of GI of different foods have been proposed to overcome the problem of variability. While these models have been accurate and reproducible, they do not take into account biological factors like differences of digestion, absorption and insulin resistance that can significantly impact GI [5]. 
In the current issue, Dharmendra et al [8] from NIN, Hyderabad, have for the first time accurately estimated the glycaemic or available carbohydrate content of different food items in vitro by carefully mimicking their digestion in the human body in controlled laboratory conditions. These values were then used to calculate the portion sizes needed for in vivo GI testing against a glucose reference as per recent guidelines [9]. Using this method, they estimated GI for a variety of foods including rice, wheat and legumes. The methods followed reliably account for the resistant starch contents in the food items also and hence are more accurate than calculations of available carbohydrate content from existing charts of total carbohydrate and fibre contents of foods. It is important to create our own database of GI of different Indian foods using acceptable and accurate methods. This study by Dharmendra et al. is an important addition in this direction. Use of standard methods will ensure that a major cause of variability of GI measurements is eliminated and we can use these values with more confidence in clinical practice.

Studies on high GI diets and risk of insulin resistance and diabetes have given inconsistent results with a few of them pointing to a higher risk $[10,11]$ while others have found no additional risk $[12,13]$. Similarly, low Gi diets have been shown to be associated with a greater reduction in $\mathrm{HbAlC}$ than with high GI diets $[14,15]$. However, the difference in $\mathrm{HbA1C}$ between the two groups is small and may not affect outcomes in the long term. Questions have also been raised on the instruments of dietary assessment that may not have been designed to capture reliably the information on GI [16]. Overall, it would appear that low GI foods can favourably affect glycaemic control in diabetic patients.

Despite all the limitations, GI continues to capture the attention of physicians and nutritionists alike as it does offer a rational way of ranking carbohydrate containing foods that has the potential to favourably affect the prevention and management of diabetes. However, one has to exercise caution and refrain from using GI as the sole basis for diabetic diet prescribing. Low GI foods like ice cream may have a high content of undesirable fat which delays carbohydrate absorption and results in lower GI but adversely affects health outcomes. GI can be used judiciously in addition to the other nutritional health needs of the patient to further enhance the quality of the food choices and improve diabetes management. RSSDI recommends that in addition to other measures, low GI foods may be incorporated [17] as healthier carbohydrate choices in diabetic diets to achieve better glycaemic control.

\section{References}

1. Jenkins DJ, Wolever TM, Taylor RH, Barker H, Fielden H, Baldwin JM, et al. Glycemic index of foods: a physiological basis for carbohydrate exchange. Am J Clin Nutr. 1981;34:362-6.

2. Brand-Miller J, Wolever TM, Foster-Powell K, Colagiuri S. The new glucose revolution. New York: Marlowe \& Company; 2003a.

3. Flint A, Moller BK, Raben A, Pedersen D, Tetens I, Holst JJ, et al. The use of glycaemic index tables to predict glycaemic index of composite breakfast meals. Br J Nutr. 2004;91:979-89.

4. Gabriele R, Angela AR, Rosalba G. Role of glycemic index and glycemic load in the healthy state, in prediabetes, and in diabetes. Am J Clin Nutr. 2008;87:269S-74S.

5. Eleazu CO. The concept of low glycemic index and glycemic load foods as panacea for type 2 diabetes mellitus; prospects, challenges and solutions. Afr Health Sci. 2016 June;16(2):468-79.

6. Davidson CJ, Zderic TW, Byerley LO, Coyle EF. Different glycemic indexes of breakfast cereals are not due to glucose entry into blood but to glucose removal by tissue. Am J Clin Nutr. 2003;78: $742-8$.

7. Venn BJ, Green TJ. Glycemic index and glycemic load: measurement issues and their effect on diet-disease relationships. Eur J Clin Nutr. 2007;61(Suppl 1):S122-31.

8. dharmendra et al. current issue of IJDDC.

9. Brouns F, Bjorck I, Frayn KN, Gibbs AL, Lang V, Slama G, et al. Glycaemic index methodology. Nutr Res Rev. 2005;18:145-71.

10. McKeown NM, Meigs JB, Liu S, Saltzman E, Wilson PW, Jacques $\mathrm{PF}$. Carbohydrate nutrition, insulin resistance, and the prevalence of the metabolic syndrome in the Framingham Offspring Cohort. Diabetes Care. 2004;27:538-46.

11. Schulze MB, Liu S, Rimm EB, Manson JE, Willett WC, Hu FB. Glycemic index, glycemic load, and dietary fiber intake and incidence of type 2 diabetes in younger and middle-aged women. Am J Clin Nutr. 2004;80:348-56.

12. Liese AD, Schulz M, Fang F, et al. Dietary glycemic index and glycemic load, carbohydrate and fiber intake, and measures of insulin sensitivity, secretion, and adiposity in the Insulin Resistance Atherosclerosis Study. Diabetes Care. 2005;28:2832-8.

13. Sahyoun NR, Anderson AL, Tylavsky AF, Lee SJ, Sellmeyer DE, Harris TB. Dietary glycemic index and glycemic load and the risk of type 2 diabetes in older adults. Am J Clin Nutr. 2008;87:126-31.

14. Brand-Miller J, Hayne S, Petocz P, Colagiuri S. Low-glycemic index diets in the management of diabetes: a meta-analysis of randomized controlled trials. Diabetes Care. 2003b;26:2261-7.

15. Opperman AM, Venter CS, Oosthuizen W, Thompson RL, Vorster HH. Meta-analysis of the health effects of using the glycaemic index in meal-planning. Br J Nutr. 2004;92:367-81.

16. Hodge AM, English DR, O'Dea K, Giles GG. Glycemic index and dietary fiber and the risk of type 2 diabetes. Diabetes Care. 2004;27: 2701-6.

17. Madhu SV, Saboo B, Makkar BM, Reddy GC, Jana J, Panda JK, et al. RSSDI clinical practice recommendations for management of type 2 diabetes mellitus. Int J Diab Dev Countries. 2015;35(1):1-71. 